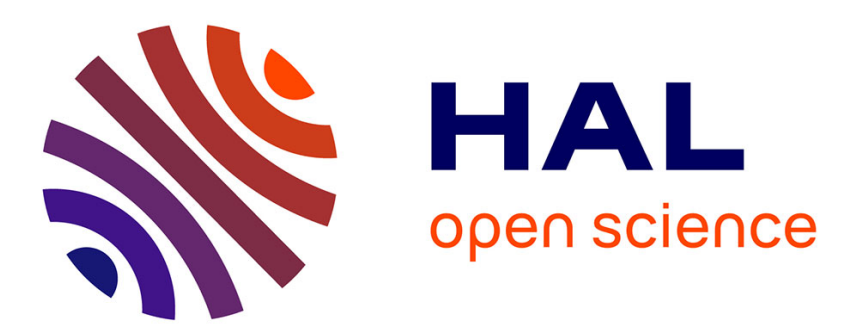

\title{
On Lexicographical Ordering in Multivariate Mathematical Morphology
}

\author{
Erchan Aptoula, Sébastien Lefèvre
}

\section{To cite this version:}

Erchan Aptoula, Sébastien Lefèvre. On Lexicographical Ordering in Multivariate Mathematical Morphology. Pattern Recognition Letters, 2008, 29 (2), pp.109-118. 10.1016/j.patrec.2007.09.011. hal00512667

\section{HAL Id: hal-00512667 \\ https://hal.science/hal-00512667}

Submitted on 31 Aug 2010

HAL is a multi-disciplinary open access archive for the deposit and dissemination of scientific research documents, whether they are published or not. The documents may come from teaching and research institutions in France or abroad, or from public or private research centers.
L'archive ouverte pluridisciplinaire HAL, est destinée au dépôt et à la diffusion de documents scientifiques de niveau recherche, publiés ou non, émanant des établissements d'enseignement et de recherche français ou étrangers, des laboratoires publics ou privés. 


\title{
On lexicographical ordering in multivariate mathematical morphology
}

\author{
E. Aptoula*, S. Lefèvre \\ Image Sciences, Computer Sciences and Remote Sensing Laboratory \\ LSIIT-UMR-7005-CNRS Louis Pasteur University, \\ Pôle API, Blvd Sébastien Brant, PO Box 10413,67412 Illkirch Cedex, France
}

\begin{abstract}
Since mathematical morphology is based on complete lattice theory, a vector ordering method becomes indispensable for its extension to multivariate images. Among the several approaches developed with this purpose, lexicographical orderings are by far the most frequent, as they possess certain desirable theoretical properties. However, their main drawback consists of the excessive priority attributed to the first vector dimension. In this paper, the existing solutions to solving this problem are recalled and two new approaches are presented. First, a generalisation of $\alpha$-modulus lexicographical ordering is introduced, making it possible to accommodate any quantisation function. Additionally, an input specific method is suggested, based on the use of a marker image. Comparative application results on colour noise reduction and texture classification are also provided.
\end{abstract}

Key words: Multivariate mathematical morphology, lexicographical ordering, colour morphology, dimension prioritisation.

\section{Introduction}

With the acceptance of complete lattice theory as the appropriate theoretical framework for mathematical morphology (MM) [11,22,23], it became possible to define morphological operators for any type of image data, as long as a complete lattice structure can be introduced on the pixel intensity range. However, as far as colour, and more generally multivariate images are concerned, the

* Corresponding author. Tel: +33(0)39024 44 99; fax: +33(0)39024 4455

Email address: aptoula@lsiit.u-strasbg.fr (E. Aptoula). 
ambiguity of ordering vectorial pixels constitutes the main obstacle in this regard. Several vector ordering mechanisms have been reported in the literature with this purpose, of which none has yet been widely accepted. A comprehensive survey on the different approaches to multivariate MM can be found in [4].

As confirmed by the last reference, among the rich variety of ordering methodologies, lexicographical ordering has been by far the most frequently employed solution with the end of extending morphological operators to multivariate image data. Its relative popularity is due mainly to its theoretical properties, since it makes it possible to totally order the underlying pixel data while also providing unique extrema. Nevertheless, lexicographical ordering suffers from a major drawback, which consists of the excessive prioritisation of the first image channel during the lexicographical cascade, hence leading to an inefficient exploitation of inter-channel relations.

In this paper, we recall the existing solutions, and present two approaches that aim to counter this inconvenience. First, a generalisation of $\alpha$-modulus lexicographical ordering is introduced, that makes it possible to quantise the first vector dimension based on an arbitrary model. Moreover, a colour morphology related example of this method is provided, using luminance and saturation dependencies. Additionally, an input specific approach is suggested, that orders the first vector components with respect to a marker image, thus providing a means of controlling the channel prioritisation of lexicographical ordering based on topological information. Both approaches are tested in the context of colour morphology, against state-of-the-art methods, with noise reduction and texture classification applications, where they provide superior results.

The rest of the paper is organised as follows. Section 2 introduces briefly the theoretical background of extending morphological operators to multivariate images as well as the problems related to lexicographical ordering and its variations. In section 3 , a generalisation of $\alpha$-modulus lexicographical ordering is presented, along with an application in colour morphology. Then, in section 4, the marker based ordering is introduced and its properties are elaborated. Next, in section 5, the results of a series of comparative tests are provided. Finally section 6 is devoted to concluding remarks.

\section{Lexicographical ordering in morphological processing}

In this section we review briefly the main issues concerning the extension of MM to multivariate images, and discuss the properties of lexicographical ordering that contributed to its widespread use, along with its inconveniences and subsequent variations. For an in-depth study of multivariate MM the 
reader can refer to the fundamental references [11,23].

\subsection{Theoretical background}

Complete lattice theory [8], is nowadays considered as the right mathematical framework for morphology [22]. The (minimal) conditions that need to be satisfied by morphological operators have been formulated by means of this theory. Specifically, introducing a complete lattice structure on the "grey levels" or intensity range of an image is sufficient for the definition of valid morphological operators.

More precisely, given an image $f: \mathcal{E} \rightarrow \mathcal{T}$ with $\mathcal{E}$ an arbitrary non empty set, a complete lattice structure is imposed on the intensity range $\mathcal{T}$. In other words, $\mathcal{T}$ must be a non empty set equipped with a partial ordering such that every non empty subset $\mathcal{P} \subseteq \mathcal{T}$ has a greatest lower bound $\wedge \mathcal{P}$ (infimum) and a least upper bound $\vee \mathcal{P}$ (supremum). Consequently, the set of images $\mathcal{F}(\mathcal{E}, \mathcal{T})$ is also a complete lattice.

For instance, in the case of grey-scale images, where usually $\mathcal{T}=\overline{\mathbb{Z}}$, or $\mathcal{T}=\overline{\mathbb{R}}$ or a finite interval $[a, b]$, the scalar order is employed for ordering the pixel values in order to obtain the lower and greater bounds. However, this becomes a much more challenging task with multivariate images, where usually $\mathcal{T}=\overline{\mathbb{Z}}^{n}$ or $\mathcal{T}=\overline{\mathbb{R}}^{n}$ with $n>1$, since there is no natural ordering relation for multivariate data.

Indeed, given a vector ranking mechanism $<$, the vectorial versions of the two fundamental morphological operators, erosion $\left(\boldsymbol{\varepsilon}_{b}\right)$ and dilation $\left(\boldsymbol{\delta}_{b}\right)$ of a multivalued image $\mathbf{f}$, by a flat structuring element (SE) $b$, can be immediately derived by means of the vectorial extrema operators $\sup _{v}$ and $\inf _{v}$ based on the given ordering:

$$
\begin{aligned}
& \boldsymbol{\varepsilon}_{b}(\mathbf{f})(\mathbf{x})=\inf _{\mathbf{s} \in b}\{\mathbf{f}(\mathbf{x}+\mathbf{s})\} \\
& \boldsymbol{\delta}_{b}(\mathbf{f})(\mathbf{x})=\sup _{\mathbf{s} \in b}\{\mathbf{f}(\mathbf{x}-\mathbf{s})\}
\end{aligned}
$$

Therefore, the main obstacle preventing the extension of morphological operators to multivalued images, consists in defining a vector ordering relation that will induce a complete lattice structure on the pixel intensity range. 


\subsection{Lexicographical ordering}

The problem of ordering multivariate data has been extensively studied in the past, especially in the field of statistics [7]. According to the ordering categorisation given in the last reference, lexicographical ordering $\left(<_{L}\right)$ is a type of conditional ordering (c-ordering), where the vector components are ordered conditionally based on some of their marginal components, and is defined as:

$$
\forall \mathbf{v}, \mathbf{v}^{\prime} \in \mathbb{R}^{n}, \quad \mathbf{v}<_{L} \mathbf{v}^{\prime} \Leftrightarrow \exists i \in\{1, \ldots, n\}, \quad\left(\forall j<i, v_{j}=v_{j}^{\prime}\right) \wedge\left(v_{i}<v_{i}^{\prime}\right)
$$

For vectors in a colour space $V W X$, where we compare first dimension $V$, then $W$ and finally $X$, we adopt the notation $V \rightarrow W \rightarrow X$.

Lexicographical ordering is particularly suitable for ordering vectors in the context of multivariate MM, in combination with image data where a natural or artificial priority order exists among the different bands, as it possesses desirable theoretical as well as practical properties. First, as all c-orderings, it is a total ordering, hence preserving the input vectors, in the contrary of marginal processing. In other words, it does not introduce any new vectors within the processing results, and thus prevents the apparition of false colours in colour morphology [26], as well as the negative effect of mixed pixel signatures during the morphological classification of multispectral images [4]. It should also be noted however that a total ordering is not always desirable within the morphological context [25].

Moreover, as it satisfies the anti-symmetry constraint, a property lacking from most reduced orderings, it makes it possible to compute unique vectorial extrema, hence effectively avoiding ambiguities during vector ordering. Furthermore, by modifying the order of comparison during the lexicographical cascade, one can easily establish different priorities among the image channels. For instance, if used in the RGB colour space, the red channel would be inevitably prioritised, whereas it would be sufficient to permute the bands as GRB to shift this priority to the green channel (figure 1).
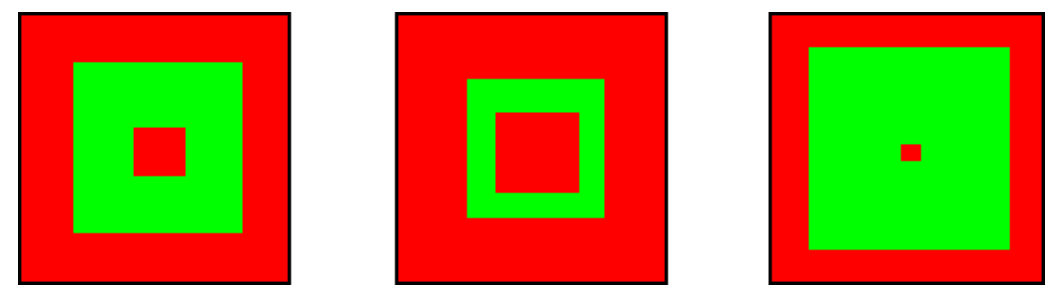

Fig. 1. Original image (left), results of applying a vectorial dilation based on a lexicographical ordering $(R \rightarrow G \rightarrow B$, middle) $(G \rightarrow R \rightarrow B$, right $)$ with a $21 \times 21$ square SE

In the case of multispectral or even hyperspectral images, where usually no a 
priori priority order exists among the tens or even hundreds of bands, the use of lexicographical ordering is practically justified only after the application of a proper transform (e.g. maximum noise fraction transform, principal components analysis, discrete cosine transform, etc) which will redistribute the total variational information contained within the bands with a monotonic order, and thus artificially create the prioritised band environment $[4,20]$. Hence making it possible to lexicographically exploit the information concentrated on the first few bands.

These invaluable properties have led to the widespread use of lexicographical ordering with the purpose of establishing multivariate morphological operators, especially in the field of colour morphology $[1,4,12,14,21]$.

\subsection{Variations of lexicographical ordering}

However, besides being equipped with several desirable properties, lexicographical ordering also suffers from a serious drawback. More precisely, the outcome of the vast majority of lexicographical comparisons, is decided based only on the first few vector components that are compared, while the remaining dimensions' contribution can be considered negligible. This property is illustrated in table 1, where the percentages of comparisons determined by the three channels of the three RGB colour images of figure 2.3, during a vectorial dilation based on a lexicographical ordering are shown. The channel occupying the first position of the lexicographical cascade (i. e. red) is obviously responsible for the vast majority of comparisons.
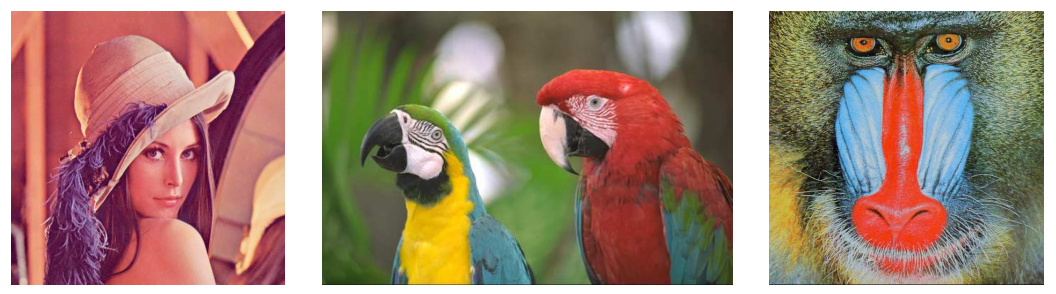

Fig. 2. The test images, from left to right, Lenna, Macaws and Mandrill.

Table 1

The percentages of comparisons that have led to equalities, and those that have been determined at each channel, during the dilation of the images of figure 2.3, with a lexicographical ordering $(R \rightarrow G \rightarrow B)$ using a square shaped SE of size $5 \times 5$ pixels.

\begin{tabular}{|c||c|c|c|c|}
\hline Images & Equalities (\%) & Red (\%) & Green (\%) & Blue (\%) \\
\hline Lenna & 0.12 & 93.02 & 6.3 & 0.56 \\
Macaws & 8.89 & 83.3 & 5.71 & 2.1 \\
Mandrill & 0.54 & 95.28 & 3.51 & 0.67 \\
\hline
\end{tabular}


Of course this might be a desired behaviour in cases where the fist image channel contains the majority of the total variational information, for instance after applying a PCA transform. Nevertheless, most often it leads to an insufficient exploitation of the image channels and inter-channel relations. This effect is most aggravated in the case of hyperspectral images, where despite the availability of hundreds of channels, only at most the first few participate in the overall process. That is why, variations of expression (3) were reported, with the end of better tuning the priority as well as degree of influence of each vector component on the comparison outcome, by means of an user defined argument $\alpha$. A list of such extensions follows.

The first attempt aiming to decrease the priority attributed to the first vector component during lexicographical comparison was made by Ortiz et al. [19], that proposed the $\alpha$-lexicographical ordering:

$$
\forall \mathbf{v}, \mathbf{v}^{\prime} \in \mathbb{R}^{n}, \quad \mathbf{v}<\mathbf{v}^{\prime} \Leftrightarrow\left\{\begin{array}{l}
v_{1}+\alpha<v_{1}^{\prime}, \text { or } \\
v_{1}+\alpha \geq v_{1}^{\prime} \text { and }\left[v_{2}, \ldots, v_{n}\right]^{T}<_{L}\left[v_{2}^{\prime}, \ldots, v_{n}^{\prime}\right]^{T}
\end{array}\right.
$$

where $\alpha \in \mathbb{R}^{+}$. The $\alpha$ argument is thus used to the end of increasing the occurrence of equivalences within the first vector dimension, since a scalar value $v_{1}$ becomes "equal" to all values contained in the interval $\left[v_{1}-\alpha, v_{1}+\alpha\right]$, hence allowing comparisons to reach more frequently the second dimension. Nevertheless, expression (4) is not transitive, and consequently does not represent an ordering from an algebraic point of view.

A further attempt was made by Aptoula and Lefèvre [6], that introduced $\alpha$-trimmed lexicographical extrema. Specifically, it can be considered as an iterative approach based on the principle of $\alpha$-trimming, where given a set $V$ of $k$ vectors, in the case of the maximum, starting from the first dimension, the contents of $V$ are sorted according to this dimension, and then the $\lceil\alpha \times k\rceil$ $(\alpha \in] 0,1])$ greatest vectors are kept with respect to the sorting order and considered as the new set $V$. By repeating this process for each dimension, at each step the initial set of vectors gets smaller, leading eventually to the desired extremum. Thus, each dimension can contribute to the computation of the extrema by means of the trimming step. For the formal description of this procedure the reader can refer to the original paper [6]. On the other hand, as this methodology is not based on a binary relation among vectors, but rather relies on a "collective" extremum computation, it does not constitute an ordering, hence leading to pseudo-morphological operators.

A theoretically sounder approach was proposed by Angulo and Serra [2] and was named $\alpha$-modulus lexicographical ordering:

$$
\forall \mathbf{v}, \mathbf{v}^{\prime} \in \mathbb{Z}^{n}, \mathbf{v}<\mathbf{v}^{\prime} \Leftrightarrow\left[\left\lceil v_{1} / \alpha\right\rceil, v_{2}, \ldots, v_{n}\right]^{T}<_{L}\left[\left\lceil v_{1}^{\prime} / \alpha\right\rceil, v_{2}^{\prime}, \ldots, v_{n}^{\prime}\right]^{T}
$$



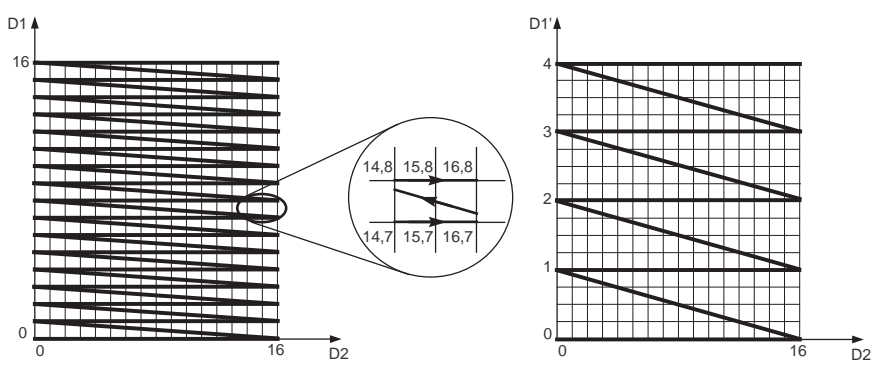

Fig. 3. Space filling curves in a bi-dimensional space for a lexicographical ordering (left), where the arrows denote the direction of increasing vectorial coordinates, and for the $\alpha$-modulus lexicographical ordering with $\alpha=4$ (right).

which aims to create equivalence groups within the first dimension. It relies on a quantisation through division by a constant $\alpha$ followed by a rounding off, which reduces the dynamic margin of the first dimension, thus allowing a greater number of comparisons to reach the second. On the contrary of the aforementioned two approaches, it is an ordering and leads to valid morphological operators.

The effect of the operation realised on the first dimension, becomes more clear by studying the space filling curves (SFC), that travel through points of multi-dimensional space. Since the search for a total vector ordering can be formulated as a search for an injective function mapping all the points of a multi-dimensional space onto an uni-dimensional space, SFC satisfy this requirement and make it possible to model different solutions, where vectors are ordered according to the position of their coordinates on the SFC. Since lexicographical ordering corresponds to a bijection [9], its SFC will pass once from all points of a bi-dimensional discrete space $D 1 \times D 2$ as illustrated in figure 3 (left), where the high priority attributed to the first dimension (D1) is represented by the high frequency of horizontal curves. Figure 3 (right) shows the quantised form $D 1^{\prime}$ of dimension $D 1$ with $\alpha=4$, which leads to the creation of equivalence groups. For instance the points $\{4,5,6,7\}$ of $D 1$ now belong to the same group 1 of $D 1^{\prime}$, and thus given two coordinates $(4,1)$ and $(7,0)$ in $D 1 \times D 2$, the first components are considered equal in $D 1^{\prime} \times D 2$ and the outcome of the comparison is determined by the second dimension, in other words $(4,1)>(7,0)$.

Nevertheless, special attention is required with the use of this ordering, since the resulting equivalence groups obviously eliminate the anti-symmetry property of lexicographical ordering. Consequently, equivalence between vectors does not occur in the usual sense within the quantised space:

$$
\forall \mathbf{v}, \mathbf{v}^{\prime} \in \mathbb{Z}^{n}, \mathbf{v}=\mathbf{v}^{\prime} \Leftrightarrow\left(\mathbf{v} \leq \mathbf{v}^{\prime}\right) \wedge\left(\mathbf{v} \geq \mathbf{v}^{\prime}\right)
$$


but rather between vectors using their original unquantised values:

$$
\forall \mathbf{v}, \mathbf{v}^{\prime} \in \mathbb{Z}^{n}, \mathbf{v}=\mathbf{v}^{\prime} \Leftrightarrow\left[v_{1}, v_{2}, \ldots, v_{n}\right]^{T}=\left[v_{1}^{\prime}, v_{2}^{\prime}, \ldots, v_{n}^{\prime}\right]^{T}
$$

In conclusion, among the solutions that have been so far reported with the purpose of fine-tuning the dimension prioritisation of lexicographical ordering, only expression (5) satisfies the theoretical requirements of an ordering and hence leads to valid morphological operators. The practical use of $\alpha$-modulus lexicographical ordering however is limited, as it relies on an implicit assumption on the dimension to be quantised. The next sections introduce a generalisation of this approach that makes it possible to employ a wider range of quantisation functions, as well as a method that relies on the, eventually morphological, smoothing of the first image channel, thus leading to equivalence groups based on spatial relations.

\section{Quantisation based $\alpha$-lexicographical ordering}

As far as its theoretical properties are concerned, $\alpha$-modulus lexicographical ordering, appears to be the most pertinent among the variations presented in section 2.3, as it provides an effective means of shifting priority away from the first vector dimension, while preserving the desirable characteristics of lexicographical ordering that have made it popular within the morphological context $[1,4,12,14,21]$.

However, its practical use relies on an important implicit assumption. More precisely, as shown in figure $4 \mathrm{a}$, the dimension under consideration is processed uniformly, based on the assumption that all of its subsets are equally "unimportant" with respect to the second dimension in the lexicographical cascade. In practice, often complicated relations are present among the image channels, and the need to shift priority to the next dimensions arises only partially. Consider for instance the case of colour morphology in the HSL colour space, where the importance of saturation is minimised for extreme levels of luminance.

In the light of these remarks, we propose the generalisation described in algorithm 1, as a replacement for the division followed by rounding off, of expression (5). Based on a simple principle, this algorithm realises a quantisation of a given discrete "pixel range" interval, in practice often [0,255], by associating to each value, a group of equivalence, the size of which is computed using a user defined function $f$, and is limited by the value of $\alpha \in \mathbb{N}^{*}$. Hence, it is possible to reformulate expression (5) as: 


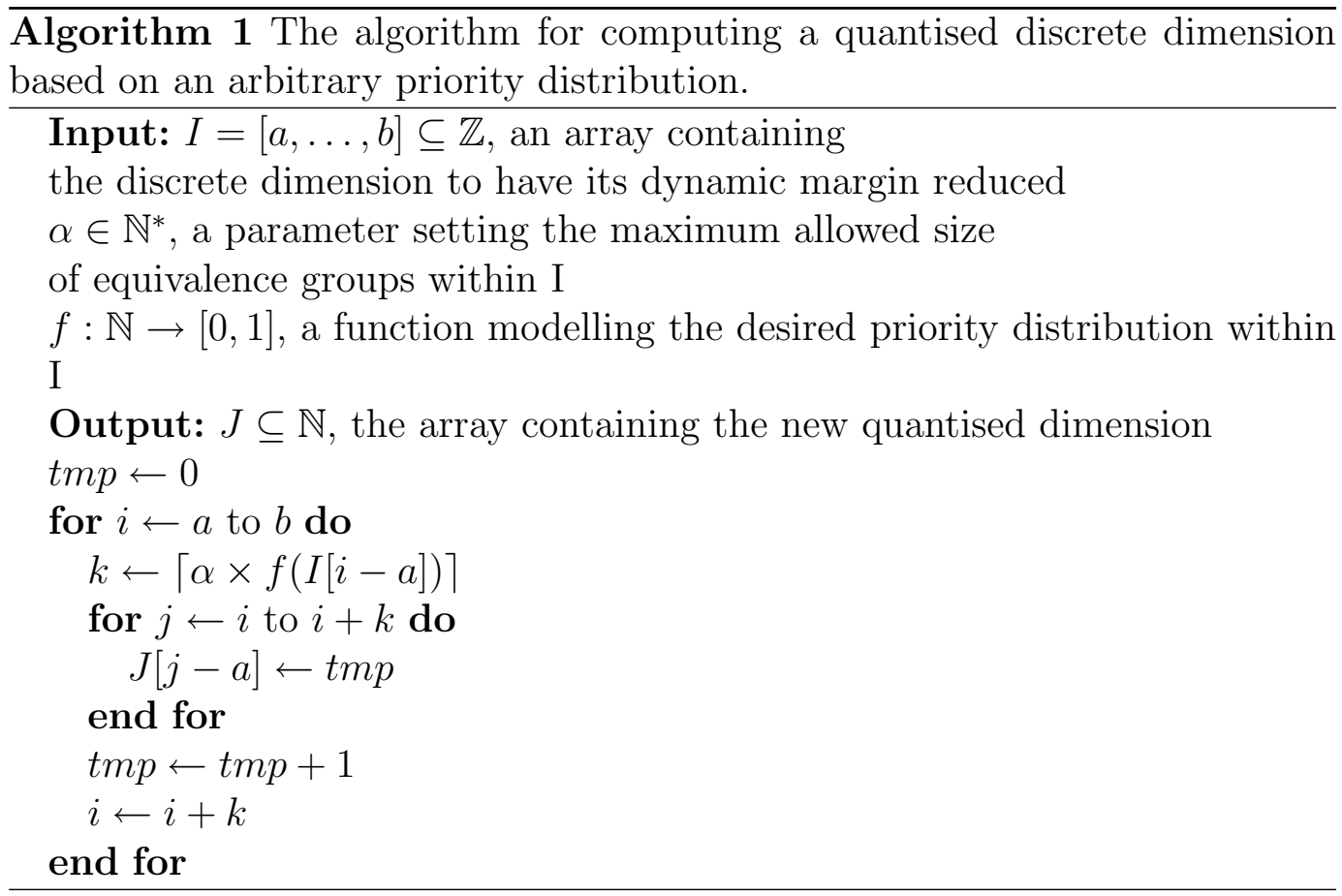

$$
\forall \mathbf{v}, \mathbf{v}^{\prime} \in \mathbb{Z}^{n}, \mathbf{v}<\mathbf{v}^{\prime} \Leftrightarrow\left[w_{1}, v_{2}, \ldots, v_{n}\right]^{T}<_{L}\left[w_{1}^{\prime}, v_{2}^{\prime}, \ldots, v_{n}^{\prime}\right]^{T}
$$

where $w_{1}$ and $w_{1}^{\prime}$ represent respectively the equivalence group of $v_{1}$ and $v_{1}^{\prime}$, obtained through algorithm 1 . Of course one is by no means limited with applying this procedure only to the first dimension. In fact, complicated interchannel relations often require the repeated use of such an approach in more than one dimensions in order to to be effectively modelled.

a)

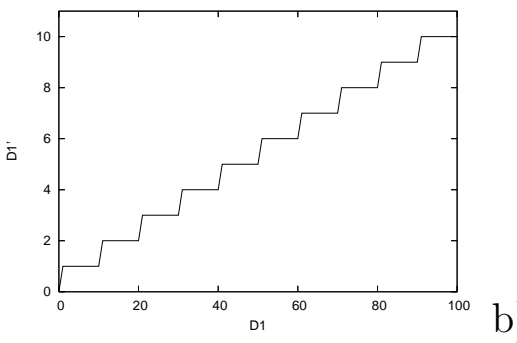

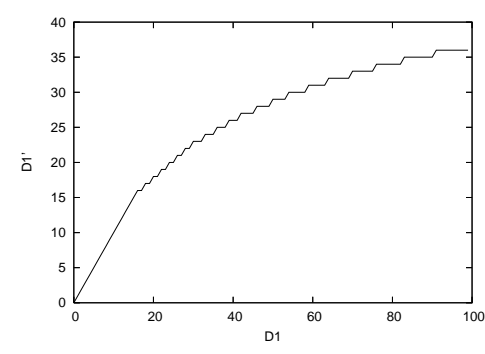

Fig. 4. Example of quantisation applied on a dimension D1 using $\alpha=10$ and expression (5) (a), and using the proposed approach with the same $\alpha$ and an exponential distribution (b).

An example of this algorithm is given in figure $4 \mathrm{~b}$, where it is applied on the integer interval $[0,100]$ using an exponential priority distribution. In other words, values close to zero are of high importance and need to be processed with a fine precision whereas the second dimension may be used with values approaching 100 . The increase in the size of equivalence groups can be easily observed as the values approach the upper interval bound. Consequently, in order to obtain the uniform distribution of figure $4 \mathrm{a}$, corresponding to the 


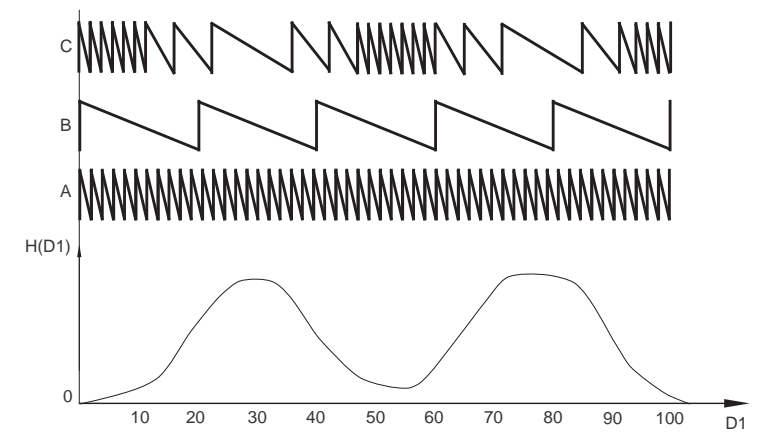

Fig. 5. The probability distribution of dimension D1 (bottom) for an arbitrary image, general appearance of the space filling curve frequency corresponding to its lexicographical ordering (A), $\alpha$-modulus lexicographical ordering with $\alpha=20$ (B), and quantisation based $\alpha$-lexicographical ordering using the dimension histogram as priority distribution function (C).

quantisation of $\alpha$-modulus lexicographical ordering, with this approach, it is sufficient to employ the constant function $\forall n \in \mathbb{N}, f(n)=1$ within algorithm 1. By means of the function $f$, once can thus model arbitrary priority distributions within image channels. Furthermore, image-specific ordering approaches may be developed using the histogram of the dimension under consideration, as shown in figure 5 , hence leading to adaptive vector orderings.

a)

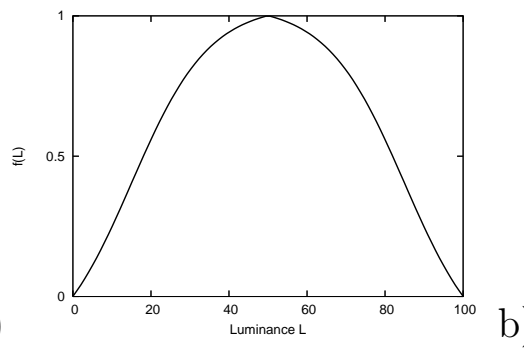

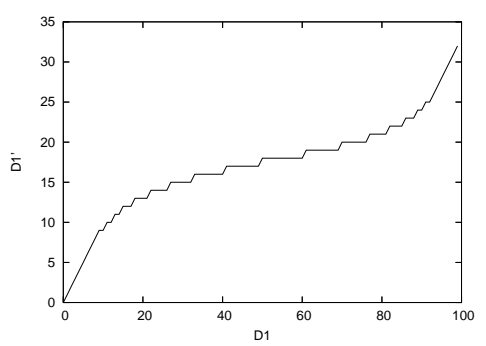

Fig. 6. The "importance" of saturation with respect to luminance in the HSL colour space with a double sigmoidal distribution (a), and the quantised form of the luminance channel using this relation (b) with $\alpha=10$ and algorithm 1.

As a more concrete example, let us return to the aforementioned case of colour morphology in the HSL colour space. Polar colour spaces exhibit an inherent prioritisation of channels, as they model the higher levels of human colour vision, and thus luminance is most often used in the first position of the lexicographical cascade when developing morphological operators $[1,4,14,19]$. However, non trivial relations are present among the three channels (luminance, saturation and hue), that are totally ignored using expression (5). Specifically, the importance of saturation is maximised for "medium" levels of luminance, whereas hue is of practical importance only for relatively high levels of saturation.

Since luminance alone is sufficient for the recognition of most objects, it oc- 
a)

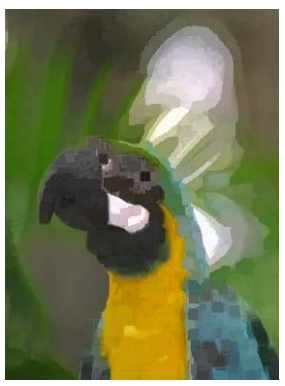

b)

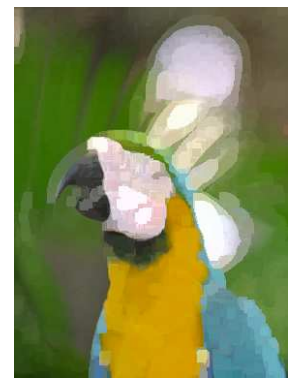

c)

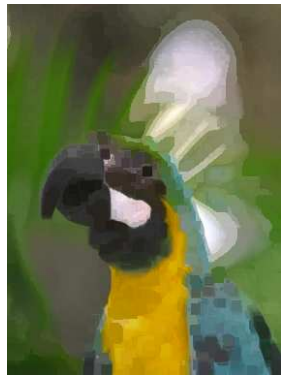

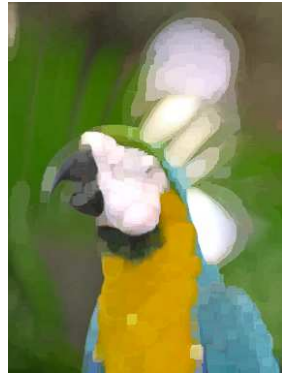

Fig. 7. The erosion and dilation results of a part of the macaws image (figure 10a) with a square shaped SE of size $9 \times 9$ pixels, using $\alpha$-modulus lexicographical ordering (5) $(\mathrm{a}, \mathrm{b})$ and the proposed quantisation based lexicographical ordering (8) along with the priority distribution illustrated in figure $6 \mathrm{a}$ and both with $\alpha=50(\mathrm{c}, \mathrm{d})$.

cupies almost always the first position of lexicographical cascades during the comparison of HSL colour vectors. For the sake of simplicity let us limit all subsequent operations on this colour space to the last two channels $(L \rightarrow S)$, since the hue, being an angular value [13] requires a reference value which would further parameterise the procedure, and besides most comparisons seldom reach the third dimension. Moreover, the same principles if desired can also be applied between saturation and hue in a similar way. The standard way for decreasing the excessive priority attributed to luminance, using $\alpha$-modulus lexicographical ordering, would lead to the quantised result shown in figure 4 a.

For illustrational purposes we choose to model the importance of saturation $f(L)$ with respect to luminance $L$ using a double sigmoidal distribution (figure $6 a)$. Consequently, by means of $f(L)$ combined with the proposed quantisation scheme, we obtain the corresponding quantised form of luminance as depicted in figure $6 \mathrm{~b}$. Observe the presence of the largest equivalence groups for medium luminance values. Hence, at this region, the number of comparisons determined by the luminance component will be minimised, accordingly to the given model, and instead will be determined by the following vector dimension, in other words saturation.

As illustrated by this relatively simple example, the proposed priority reduction scheme constitutes a generalisation of the quantisation step in expression (5), that provides a high degree of flexibility, making it possible for the user to accommodate within the resulting ordering relation practically any particular model of priority distribution. An application example is given in figure 7 , which shows the results obtained after dilation and erosion with orderings (5) and (8) using the same value of $\alpha$. The differences between the two approaches are fairly visible, in particular at the bleak and head region, where more smoothed variations are obtained. 


\section{Marker based lexicographical ordering}

Creating artificial equivalence groups within the dimension to have its lexicographical priority reduced, is an effective means of shifting priority to the remaining vector dimensions. However, this is an operation realised independently from the images to be processed (except if the priority distribution is image specific, e.g. based on its histogram), and no a priori information is available on where the, eventually abrupt, equivalence group limits correspond within the input image. This undesirable situation is illustrated in figure 8 where the equivalence groups of an uni-dimensional discrete signal, obtained with expression (5), lead to artificial edges/value variations during comparison.

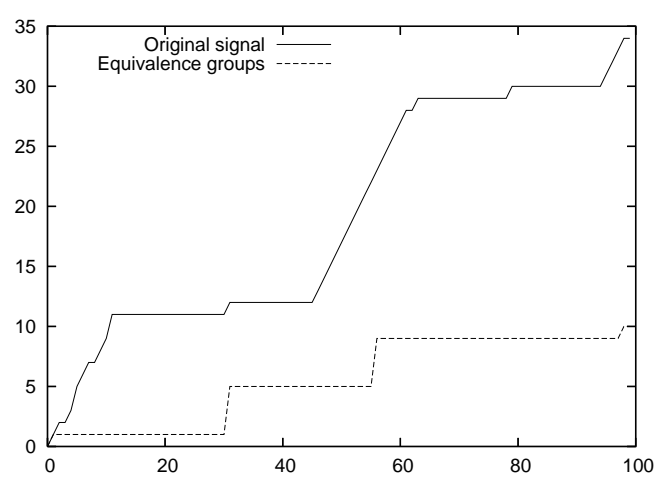

Fig. 8. Example of an uni-dimensional discrete signal with its corresponding equivalence groups obtained with expression (5) and $\alpha=11$.

An alternative way of achieving a priority shift that avoids this inconvenience, consists in assuming an image specific approach and forming these equivalence groups based on the content of the image to be processed. Specifically, one can preprocess the input image's channel that is to have its priority reduced, for instance the luminance channel in the example of section 3 , so that it is "flattened", or in other words heavily smoothed. If we call this image a "marker" $m: \mathbb{Z}^{2} \rightarrow \mathbb{R}$, then the ordering of vectors of image $g$ can be realised as follows:

$$
\begin{aligned}
& \forall x, y, s, t \in \mathbb{Z}, \quad g(x, y)<g(s, t) \Leftrightarrow \\
& \quad\left[m(x, y), g_{2}(x, y), \ldots, g_{n}(x, y)\right]^{T}<_{L}\left[m(s, t), g_{2}(s, t), \ldots, g_{n}(s, t)\right]^{T}
\end{aligned}
$$

In other words, the formation of equivalence groups is now controlled only by the marker image. If a couple of pixels have the same value in the marker image, then they are considered equal for the dimension that this marker image represents, and the comparison outcome is determined by the following dimensions. Hence equivalence groups are now formed at the flat regions of $m$ (figure 9). The "flattening" process may be achieved using a variety of filters 


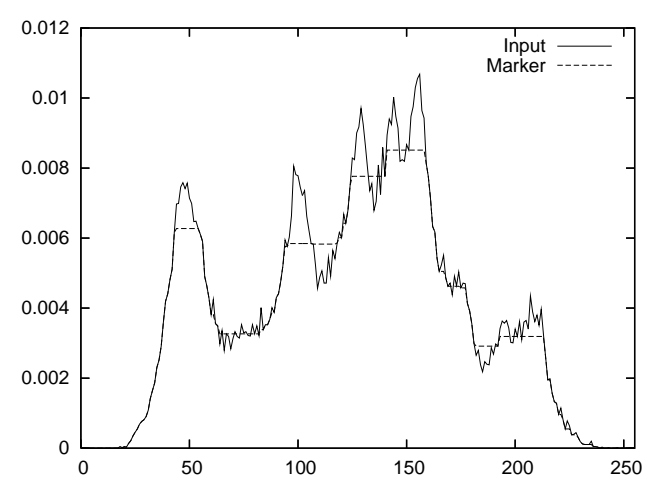

Fig. 9. Example of an uni-dimensional signal and its marker, obtained with an alternating sequential filter using line shaped SEs of length 5 pixels.

such as large median filters, alternating sequential filters [24], morphological levelings [17], etc. As an application example, let us return to the HLS colour space, and consider a lexicographical ordering of type $L \rightarrow S$. As such the luminance component would determine the outcome of the vast majority of comparisons. Shifting priority to the second dimension (i. e. saturation) can be achieved using for instance the quantisation example of the previous section. However, since edge related information is represented in its majority at the luminance component, it is obviously pertinent to employ this component at object edges and borders, while using saturation for homogeneous or flat image regions. This relatively constrained situation can be resolved with expression (9) as shown in figure 10.

More precisely, the priority shift towards saturation can be effectively limited with only the relatively homogeneous regions of the input, by flattening them using a morphological levelling applied to the luminance component. The "priority map" shown in figure 10d asserts this statement. Specifically, this map associates to each pixel the percentage of comparisons within the SE that have been determined using the first dimension (i. e. luminance). Hence dark

a)

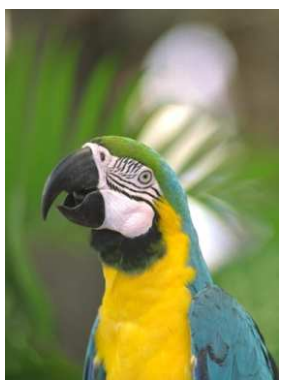

b)

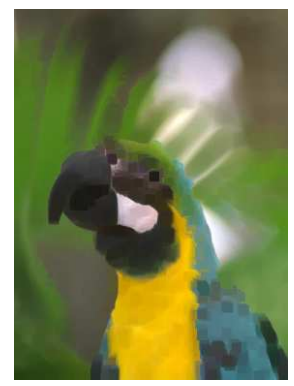

c)

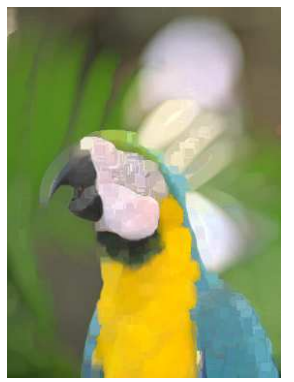

d)

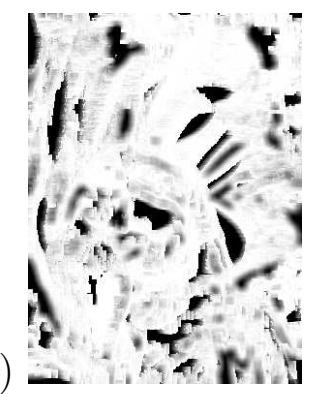

Fig. 10. The original image (a), its erosion (b) and dilation (c) results with a square shaped SE of size $9 \times 9$ pixels, using the marker based ordering (9) along as marker its morphological levelling. The marker of the levelling has been obtained by means of alternating sequential filters using the same SE. And rightmost, is the priority map (d). 
areas correspond to pixels were the outcome of vector comparisons has been determined using the saturation component, whereas bright areas represent pixels were luminance, the first vector dimension, has been used to order the vectors.

Consequently, although the creation of equivalence groups is image specific, and needs to be realised independently for each image to be processed, marker based ordering provides nonetheless a means of avoiding the artificial edge related pitfalls of the aforementioned approaches, by taking into account the spatial relationships of pixels, thus making it possible to accommodate topological restrictions during ordering.

\section{Applications}

In this section, the proposed ordering approaches (8) (QuantLex) and (9) (MarkerLex) are compared against the $\alpha$-modulus (AmodLex) (5) as well as the standard lexicographical ordering (Lexico) (3). For the sake of objectivity two different applications are employed, both using colour images, noise reduction and texture classification. The other variations (i. e. $\alpha$-trimmed and $\alpha$-lexicographical) were omitted since they are not orderings and hence do not verify fundamental theoretical requirements. Both AmodLex and QuantLex are set with $\alpha=10$, the image data being in the HSL colour space and in the integer interval $[0,255]$. Once more, for the reasons given in section 3 an ordering ignoring the hue $(L \rightarrow S)$ is employed. Specifically, the priority distribution depicted in figure $6 \mathrm{a}$ is used for QuantLex. As to MarkerLex, the marker image is obtained by levelling $(\lambda=3)$ the opening-closing result of the luminance band employing a square shaped SE of size $7 \times 7$ pixels.

\subsection{Colour noise reduction}

For the first application the 100 test images of the Berkeley segmentation dataset have been employed [16]. The image data has been corrupted in the RGB colour space with uncorrelated $(\sigma=32, \rho=0.0)$ zero-mean Gaussian noise, and in order to quantify the relative performances, the relative normalized mean squared error (RNMSE) has been calculated, also in RGB:

$$
\operatorname{RNMSE}=\frac{\sum_{x, y}\left\|f(x, y)-f_{f}(x, y)\right\|^{2}}{\sum_{x, y}\left\|f(x, y)-f_{n}(x, y)\right\|^{2}}
$$

where $f(x, y), f_{n}(x, y), f_{f}(x, y)$ denote the vector pixels at position (x,y) respectively of the original, the noisy and filtered images, while $\|\cdot\|$ represents 
the Euclidean norm. The images have been filtered using a square shaped SE of size $3 \times 3$ pixels and the open-close close-open filter $(\mathrm{OCCO})$ :

$$
\operatorname{OCCO}(f)=\frac{1}{2} \phi(\gamma(f))+\frac{1}{2} \gamma(\phi(f))
$$

where $\phi$ and $\gamma$ denote respectively the vectorial closing and opening operators. Table 2

The average $1000 \times R N M S E$ errors against uncorrelated Gaussian noise $(\sigma=$ $32, \rho=0.0)$ using the test images of the Berkeley segmentation dataset.

\begin{tabular}{|c|c|c|c|c|}
\hline Luminance & Lexico & AmodLex & QuantLex & MarkerLex \\
\hline 565.63 & 565.59 & 568.65 & $\mathbf{5 6 4 . 1 9}$ & 651.47 \\
\hline
\end{tabular}

The average error rates are given in table 2. One can easily remark the extremely similar values obtained by using only the luminance channel for comparisons and the standard lexicographical ordering. A result which asserts the highly asymmetrical priority attributed to the first vector component. Moreover, the transition of this priority to the saturation band by means of the AmodLex ordering does not result in an improvement, leading us to assume that the luminance channel is more pertinent for this task. However, the proposed QuantLex approach gives the overall best error ratio, though only slightly superior than its lexicographical counterpart, by exploiting the inner relationships among luminance and saturation. As to MarkerLex, it is obviously not suitable for the task of noise reduction, since its highly smoothed marker image prevents it from accessing the fine differences among the corrupted pixels of the luminance band.

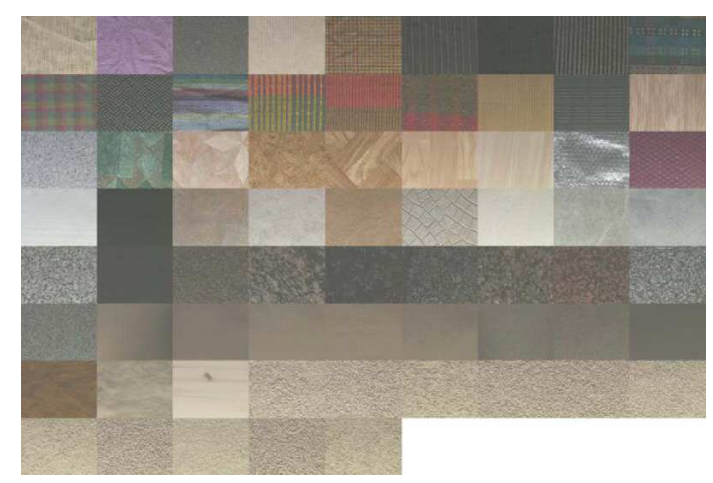

Fig. 11. Examples of the 68 colour textures of Outex13 [15].

\subsection{Colour texture classification}

As to texture classification, it has been chosen to employ the publicly available Outex13 colour texture database (figure 11) $[15,18]$. The descriptors have been obtained by means of the morphological version of the autocorrelation operator, namely morphological covariance, which represents the family of joint 
methods for the processing of colour and textural information $[3,10]$. The vectorial version of morphological covariance $K^{\prime}$ of an image $f$, is defined as the volume Vol of the image (i. e. sum of pixel values), eroded by a pair of points $P_{2, v}$ separated by a vector $\mathbf{v}$ :

$$
K^{\prime}\left(f ; P_{2, v}\right)=\operatorname{Vol}\left(\varepsilon_{P_{2, v}}(f)\right)
$$

In practice, $K^{\prime}$ is computed for varying lengths of $\mathbf{v}$, and most often as also here the normalised version $K$ is used for measurements:

$$
K(f)=\operatorname{Vol}\left(\varepsilon_{P_{2, v}}(f)\right) / \operatorname{Vol}(f)
$$

The resulting $K$ series provides information on the periodicity and coarseness of its input $[5,24]$. Whereas additional information concerning the anisotropy of the textures can be obtained by plotting against not only different lengths of $\mathbf{v}$, but orientations as well.

The covariance based feature vectors have been calculated using four directions for the point pairs $\left(0^{\circ}, 45^{\circ}, 90^{\circ}, 135^{\circ}\right)$, each along with distances ranging from 1 to 49 pixels in steps of size two. Consequently 25 values are available for each direction, making a total of 100 values for every image channel after concatenation. The classification process has been realised using a $\mathrm{kNN}$ classifier $(k=1)$ along with the Euclidean distance.

Table 3

Classification rates in $\%$ for the textures of Outex13, using vectorial erosion based covariance.

\begin{tabular}{|c|c|c|c|c|}
\hline Luminance & Lexico & AmodLex & QuantLex & MarkerLex \\
\hline 76.03 & 76.05 & 76.16 & 77.17 & $\mathbf{7 9 . 8 6}$ \\
\hline
\end{tabular}

The classification rates are shown in table 3. As before, one can immediately remark the almost identical results of Lexico and Luminance. Once more the quantisation based lexicographical ordering leads to an improvement over AmodLex, as it controls the transitions among the image channels in a finer fashion, the difference being however almost negligible (1.01 points). Contrarily to noise reduction, the marker based approach provides the best results in this case, and outperforms the $\alpha$-modulus lexicographical ordering by 3.7 points. Apparently, the use of saturation with relatively "flat" regions, and that of luminance with transitions within the image, lead to more pertinent descriptors by better combining colour and textural information. 


\section{Conclusion}

Lexicographical ordering has proven to be an invaluable vector ordering methodology for MM, as it possesses multiple desirable theoretical properties and makes it possible to easily customize the order of comparison among image channels. Its widespread use however is mostly hindered by the usually excessive priority attributed to the first vector dimension. Among the existing approaches countering this problem that have been recalled, $\alpha$-modulus lexicographical ordering stands out as the most theoretically viable solution.

In this paper, two additional solutions have been presented. First, a generalisation to $\alpha$-modulus lexicographical ordering has been proposed, avoiding the implicit assumption of a constant priority distribution, hence making it possible to model arbitrarily complicated inter-channel priority relations. The inconvenience of quantisation based approaches however, is the introduction of artificial edges at equivalence group borders, as shown in section 4 . One possible way of avoiding this effect is to follow an image specific approach, where the priority shift occurs according to topological and spatial criteria.

The interest of the proposed approaches has been illustrated by means of comparative applications in the context of colour morphology. The quantisation based ordering has systematically outperformed $\alpha$-modulus lexicographical ordering in noise reduction, by using a finer tuned priority transition among the image channels. As to the marker based ordering, it has provided the overall best results in terms of texture classification accuracy by limiting the use of saturation to homogeneous image regions while using luminance for edges and object transitions, hence leading to more pertinent descriptors.

Future work will concentrate on the study of the theoretical properties of the proposed approaches as well as on their more application oriented use.

\section{Acknowledgements}

The authors wish to thank the anonymous reviewers for their valuable comments.

\section{References}

[1] J. Angulo, Unified morphological color processing framework in a lum/sat/hue representation, in: C. Ronse, L. Najman, E. Decencière (eds.), Proceedings of 
the 7th ISMM, vol. 30 of Computational Imaging and Vision, Springer-Verlag, Dordrecht, Germany, 2005.

[2] J. Angulo, J. Serra, Morphological coding of color images by vector connected filters, in: IEEE Proceedings of the 7th International Symposium on Signal Processing and Its Applications (ISSPA'2003), vol. 1, Paris, France, 2003.

[3] E. Aptoula, S. Lefèvre, Spatial morphological covariance applied to texture classification, in: Proceedings of the International Workshop on Multimedia Content Representation, Classification and Security, vol. 4105, Istanbul, Turkey, 2006 .

[4] E. Aptoula, S. Lefèvre, A comparative study on multivariate mathematical morphology, Pattern Recognition(doi:10.1016/j.patcog.2007.02.004).

[5] E. Aptoula, S. Lefèvre, On morphological color texture characterization, in: International Symposium on Mathematical Morphology, Rio de Janeiro, Brazil, 2007.

[6] E. Aptoula, S. Lefèvre, Pseudo multivariate morphological operators based on $\alpha$-trimmed lexicographical extrema, in: IEEE Proceedings of the International Symposium on Image and Signal Processing and Analysis, Istanbul, Turkey, 2007.

[7] V. Barnett, The ordering of multivariate data, Journal of the Statistical Society A 139 (3) (1976) 318-355.

[8] G. Birkhoff, Lattice Theory, American Mathematical Society, Providence, 1967.

[9] J. Chanussot, P. Lambert, Extending mathematical morphology to color image processing, in: International Conference on Color in Graphics and Image Processing, Saint-Etienne, France, 2000.

[10] I. Epifanio, P. Soille, Morphological texture features for unsupervised and supervised segmentations of natural landscapes, IEEE Transactions on Geosciences and Remote Sensing 45 (4) (2007) 1074-1083.

[11] J. Goutsias, H. J. A. M. Heijmans, K. Sivakumar, Morphological operators for image sequences, Computer Vision and Image Understanding 62 (3) (1995) $326-346$.

[12] A. Hanbury, J. Serra, Mathematical morphology in the HLS colour space, in: T. Cootes, C. Taylor (eds.), 12th British Machine Vision Conference, Manchester, UK, 2001.

[13] A. Hanbury, J. Serra, Morphological operators on the unit circle, IEEE Transactions on Image Processing 10 (12) (2001) 1842-1850.

[14] G. Louverdis, M. Vardavoulia, I. Andreadis, P. Tsalides, A new approach to morphological color image processing, Pattern Recognition 35 (8) (2002) 17331741. 
[15] T. Mäenpää, M. Pietikäinen, Classification with color and texture: jointly or separately?, Pattern Recognition 37 (8) (2004) 1629-1640.

[16] D. Martin, C. Fowlkes, D. Tal, J. Malik, A database of human segmented natural images and its application to evaluating segmentation algorithms and measuring ecological statistics, in: Proceedings of the 8th International Conference on Computer Vision, vol. 2, Vancouver, Canada, 2001.

URL

http://www.cs.berkeley.edu/projects/vision/grouping/segbench/

[17] F. Meyer, Levelings, image simplification filters for segmentation, Journal of Mathematical Imaging and Vision 20 (2004) 59-72.

[18] T. Ojala, T. Mäenpää, M. Pietikäinen, J. Viertola, J. Kyllönen, S. Huovinen, Outex: New framework for empirical evaluation of texture analysis algorithms, in: Proceedings of the 16th ICPR, vol. 1, Quebec City, Canada, 2002.

[19] F. Ortiz, F. Torres, J. Angulo, S. Puente, Comparative study of vectorial morphological operations in different color spaces, Proceedings of Intelligent robots and computer vision XX: Algorithms, Techniques, and Active Vision 4572 (2001) 259-268.

[20] A. Plaza, P. Martinez, R. Perez, J. Plaza, A new approach to mixed pixel classification of hyperspectral imagery based on extended morphological profiles, Pattern Recognition 37 (6) (2004) 1097-1116.

[21] J. Rivest, Morphological operators on complex signals, Signal Processing 84 (1) (2004) 133-139.

[22] C. Ronse, Why mathematical morphology needs complete lattices, Signal Processing 21 (2) (1990) 129-154.

[23] J. Serra, Anamorphoses and function lattices, in: E. R. Dougherty (ed.), Mathematical Morphology in Image Processing, chap. 13, Marcel Dekker, New York, 1993, pp. $483-523$.

[24] P. Soille, Morphological Image Analysis : Principles and Applications, SpringerVerlag, Berlin, 2003.

[25] P. Soille, Beyond self-duality in morphological image analysis, Image Vision and Computing 23 (2) (2005) 249-257.

[26] H. Talbot, C. Evans, R. Jones, Complete ordering and multivariate mathematical morphology, in: H. Heijmans, J. Roerdink (eds.), Mathematical Morphology and Its Applications to Image and Signal Processing, Kluwer Academic Press, Amsterdam, 1998. 